\title{
Natural killer-like signature observed post therapy in locally advanced rectal cancer is a determinant of pathological response and improved survival
}

Matthew Alderdice ${ }^{1}$, Philip D Dunne ${ }^{2}$, Aidan J Cole ${ }^{2,3}$, Paul G O’Reilly ${ }^{2}$, Darragh G McArt ${ }^{1}$, Vicky Bingham $^{1}$, Marc-Aurel Fuchs ${ }^{1}$, Stephen McQuaid ${ }^{1}$, Maurice B Loughrey ${ }^{2,4}$, Graeme I Murray ${ }^{5}$, Leslie M Samuel ${ }^{6}$, Mark Lawler ${ }^{2}$, Richard H Wilson ${ }^{2,3}$, Manuel Salto-Tellez ${ }^{1,2,4,7}$ and Vicky M Coyle M $^{2,3,7}$

${ }^{1}$ Northern Ireland Molecular Pathology Laboratory, Centre for Cancer Research and Cell Biology, Queens University Belfast, Belfast, Northern Ireland; ${ }^{2}$ Centre for Cancer Research and Cell Biology, Queens University Belfast, Belfast, Northern Ireland; ${ }^{3}$ Cancer Centre, Belfast Health and Social Care Trust, Belfast, Northern Ireland; ${ }^{4}$ Department of Tissue Pathology, Royal Victoria Hospital, Belfast Health and Social Care Trust, Belfast, Northern Ireland; ${ }^{5}$ Department of Pathology, School of Medicine, Medical Sciences and Nutrition, University of Aberdeen, Aberdeen, UK and ${ }^{6}$ Department of Clinical Oncology, Aberdeen Royal Infirmary, NHS Grampian, Aberdeen, UK

Around $12-15 \%$ of patients with locally advanced rectal cancer undergo a pathologically complete response (tumor regression grade 4) to long-course preoperative chemoradiotherapy; the remainder exhibit a spectrum of tumor regression (tumor regression grade 1-3). Understanding therapy-related transcriptional alterations may enable better prediction of response as measured by progression-free and overall survival, in addition to aiding the development of improved strategies based on the underlying biology of the disease. To this end, we performed high-throughput gene expression profiling in $\mathbf{4 0}$ pairs of formalin-fixed paraffin-embedded rectal cancer biopsies and matched resections following long-course preoperative chemoradiotherapy (discovery cohort). Differential gene expression analysis was performed contrasting tumor regression grades in resections. Enumeration of the tumor microenvironment cell population was undertaken using in silico analysis of the transcriptional data, and real-time PCR validation of NCR1 undertaken. Immunohistochemistry and survival analysis was used to measure CD56+ cell populations in an independent cohort $(n=150)$. Gene expression traits observed following long-course preoperative chemoradiotherapy in the discovery cohort suggested an increased abundance of natural killer cells in tumors that displayed a clinical response to CRT in a tumor regression grade-dependent manner. CD56+ natural killer-cell populations were measured by immunohistochemistry and found to be significantly higher in tumor regression grade 3 patients compared with tumor regression grade 1-2 in the validation cohort. Furthermore, it was observed that patients positive for CD56 cells after therapy had a better overall survival $\left(\mathrm{HR}=0.282,95 \% \mathrm{Cl}=0.109-0.729, X^{2}=7.854, P=0.005\right)$. In conclusion, we have identified a novel post-therapeutic natural killer-like transcription signature in patients responding to long-course preoperative chemoradiotherapy. Furthermore, patients with a higher abundance of CD56-positive natural killer cells post long-course preoperative chemoradiotherapy had better overall survival. Therefore, harnessing a natural killer-like response after therapy may improve outcomes for locally advanced rectal cancer patients. Finally, we hypothesize that future assessment of this natural killer-like response in on-treatment biopsy material may inform clinical decision-making for treatment duration.

Modern Pathology (2017) 30, 1287-1298; doi:10.1038/modpathol.2017.47; published online 16 June 2017

Correspondence: Professor M Salto-Tellez, MD-LMS, FRCPath, FRCP, Centre for Cancer Research and Cell Biology Queens University Belfast, 97 Lisburn Road, Belfast BT9 7BL, Northern Ireland.

E-mail: m.salto-tellez@qub.ac.uk

${ }^{7}$ These authors contributed equally to this work.

Received 6 February 2017; revised 27 March 2017; accepted 29

March 2017; published online 16 June 2017
Long-course preoperative chemoradiotherapy for the treatment of locally advanced rectal cancer typically consists of a fluoropyrimidine (5-fluorouracil or capecitabine) in combination with 45-50.4 Grays of pelvic radiation over $5-5.5$ weeks in 1.8 Gy fractions with an interval of 8-12 weeks before total 
mesorectal excision. Following long-course preoperative chemoradiotherapy, $12-15 \%$ of patients experience a pathologically complete response. ${ }^{1}$ The remaining locally advanced rectal cancer patients exhibit a full spectrum of response from minimal residual malignancy to progressive disease. There are at least six published classification systems for locally advanced rectal cancer tumor regression. ${ }^{2}$ However, many institutions have adopted the four-tier tumor regression grade system similar to that published by Rodel et al, ${ }^{3}$ due to its improved ability to predict prognosis and scoring concordance between pathologists. The extent of tumor regression after therapy has previously been associated with disease-free survival, ${ }^{3}$ therefore, an increased understanding of the biology that mediates tumor regression is crucial. In addition, identifying therapy-related alterations to the tumor microenvironment architecture may provide guidance for alternative therapies for patients with poor outcomes. Modulation of key components of the immune system have been observed in a variety of cancers treated preoperatively with radiotherapy and chemoradiotherapy, including sarcoma and rectal cancer. With regards to rectal cancer, increased prevalence of CD3, CD4, and CD8 cell populations have been observed after long-course preoperative chemoradiotherapy. ${ }^{4-7}$ Based on these findings, we hypothesize that the transcriptome of posttherapeutic tumor specimens and the tumor microenvironment will be representative of specific biology and thus may direct us to predictors of response to therapy.

\section{Materials and methods}

\section{Clinical Specimens, Specimen Annotation, Nucleic Acid Extraction}

Ethical permission for the study was authorized by the Northern Ireland Biobank (Research Ethics Committee number-16/NI/0030. Project code: NIB13-0103). Sixty consecutive patients were identified who received long-course preoperative chemoradiotherapy for locally advanced rectal cancer. Where available, formalin-fixed paraffin-embedded diagnostic biopsies $(n=40)$ and the corresponding rectal resections $(n=40)$ specimens were obtained from these patients. All patients received longcourse CRT comprising $45 \mathrm{~Gy} / 25$ fractions over 5 weeks along with 5-fluorouracil or capecitabine. Patients underwent total mesorectal excision at an interval of 1-14 weeks (mean=9) following treatment. The clinical and pathological details of the patients are summarized in Supplementary Table 1. Tumor regression grade was scored using a four-tier system. Tumor regression grade 1 patients experience minor regression, tumor regression grade 2 patients experience significant regression, tumor regression grade 3 patients will have only small groups or single remaining malignant glands and tumor regression grade 4 will have no residual tumor after therapy. All specimens have been assessed by a standardized protocol, whereby, if no tumor is evident macroscopically, then the site of tumor, and any scars of areas of ulceration, are processed in entirety for histological examination. Tumor regression grading was carried out by a colorectal specialist pathologist (ML). H\&E stains of each specimen were annotated by a pathologist (MS-T) enriching for the epithelial compartment of both biopsy and resection, while including the fibroinflammatory component immediately adjacent to the epithelial component.

Total RNA was extracted using the RNeasy formalin-fixed paraffin-embedded kit (Qiagen) and genomic DNA was extracted using the DNeasy formalin-fixed paraffin-embedded kit (Qiagen). Integrity and purity of nucleic acids was assessed using the Nanodrop 2000 (ThermoFisher Scientific). Nucleic acids were quantified using the Qubit RNA HS Assay kit (ThermoFisher Scientific) and TaqMan RNase P Detection kit (ThermoFisher).

\section{Whole-Genome DASL Array and Real-Time (RT)-PCR}

Gene expression profiling was conducted on the Whole-Genome Gene expression DASL (cDNAmediated, annealing, selection and ligation) highthroughput assay (Illumina) as reported elsewhere. ${ }^{8}$ The RNA concentrations are detailed in Supplementary table 2 and the total input of RNA for each array was approximately 50ng as quantified by Qubit (Thermofisher Scientific). These data are available via gene expression omnibus-accession GSE94104. Transcripts of interest were validated in samples where there was sufficient RNA remaining using Roche RealTime ready catalog and designer assays; NCR1 (1000106257), SPANXA1 (100106293), GAPDH (100107995), and B2M (100108004). Reverse-transcribed cDNA was generated using the First strand transcriptor kit (Roche) and pre-amplifed using the cDNA pre-amp kit (Roche) and assayed on the Roche Lightcycler 480 II. Suitable housekeeping genes were selected after assaying gene expression stability on a biopsy and tumor regression grade 1-3 resection specimens using the Human Reference Gene Panel (Roche). Gene expression fold changes ( $\Delta \Delta \mathrm{Cp}$ values) were calculated using the Second derivative method max instead of the fit point method for accurate quantification of low gene expression levels. ${ }^{9}$

\section{Immunohistochemistry}

All H\&E stains were performed using the Sakura Autostainer. Using the Bond Max (Leica microsystems), immunohistochemistry was performed on formalin-fixed paraffin-embedded sections (3 $\mu \mathrm{m}$ thick) and stained with CD56 antibody 
NCL-L-CD56-1B6 (Novocastra) at a 1:100 dilution. Digital images were taken using the Aperio ScanScope ( $\times 40$ magnification). Patients were considered to be CD56 positive if a single core had $\geq 4$ positive cells; adopted from a CD56 immunohistochemistry tissue microarray scoring system published by Sconnochia et al. ${ }^{10}$

\section{Tissue Microarray}

Ethical permission was granted to investigate a posttherapeutic rectal cancer tissue microarray provided by the Grampian Biorepository as a validation cohort (North of Scotland research ethics committee number-11/NS/0015, Tissue request number TR71). The 150 patient resections were collected between 2005 and 2011 and all patients had long-course chemoradiotherapy (45 Gy) and concurrent capecitabine (825 mg/m $\mathrm{m}^{2}$ twice daily) as preoperative treatment. ${ }^{11}$ The tissue microarray was constructed using two $1 \mathrm{~mm}\left(0.79 \mathrm{~mm}^{2}\right)$ cores from each patient resection. The tumor regression grading of these samples was conducted using a similar classification system (complete response, good partial, partial, and minimal) by an expert gastrointestinal pathologist (GIM) allowing an easy conversion into the tumor regression grade 1-4 classification system adopted in our own study. The clinical and pathological details of the patients in this cohort are summarized in Supplementary Table 3. Patient all-cause mortality was identified from interrogation of the electronic hospital patient information system where status (alive/dead) was recorded. At the time of censoring of patient outcome data, there had been $35(23.5 \%)$ deaths. The mean patient survival was 72 months (95\% confidence interval (CI) 68-75 months). Clinical follow-up was a minimum of 19 months up to maximum of 99 months.

\section{Exclusion Criteria and Comparative Assessment}

The inclusion and exclusion process of patients in the discovery and validation cohorts is outlined in the consort diagram in Figure 1. All tumor regression grade 4 patients were excluded from the discovery cohort $(n=5)$, as were patients that tumor had been exhausted by serial sectioning $(n=12)$. A further three patients failed to produce transcriptional profiles. Similarly, for the validation cohort, tumor regression grade 4 patients were excluded from subsequent analysis $(n=73)$ as were cores that lacked tumor with excessive fibrosis, muscle and mucin $(n=98)$ which was in line with the approach for transcriptional profiling of NIB13-0103 cohort.

Comparative assessment of clinical and pathological parameters of both patient cohorts demonstrated their suitability as clinically representative discovery and validation cohorts for investigating tumor regression in locally advanced rectal cancer. Patient specimens were obtained for the NIB13-0103
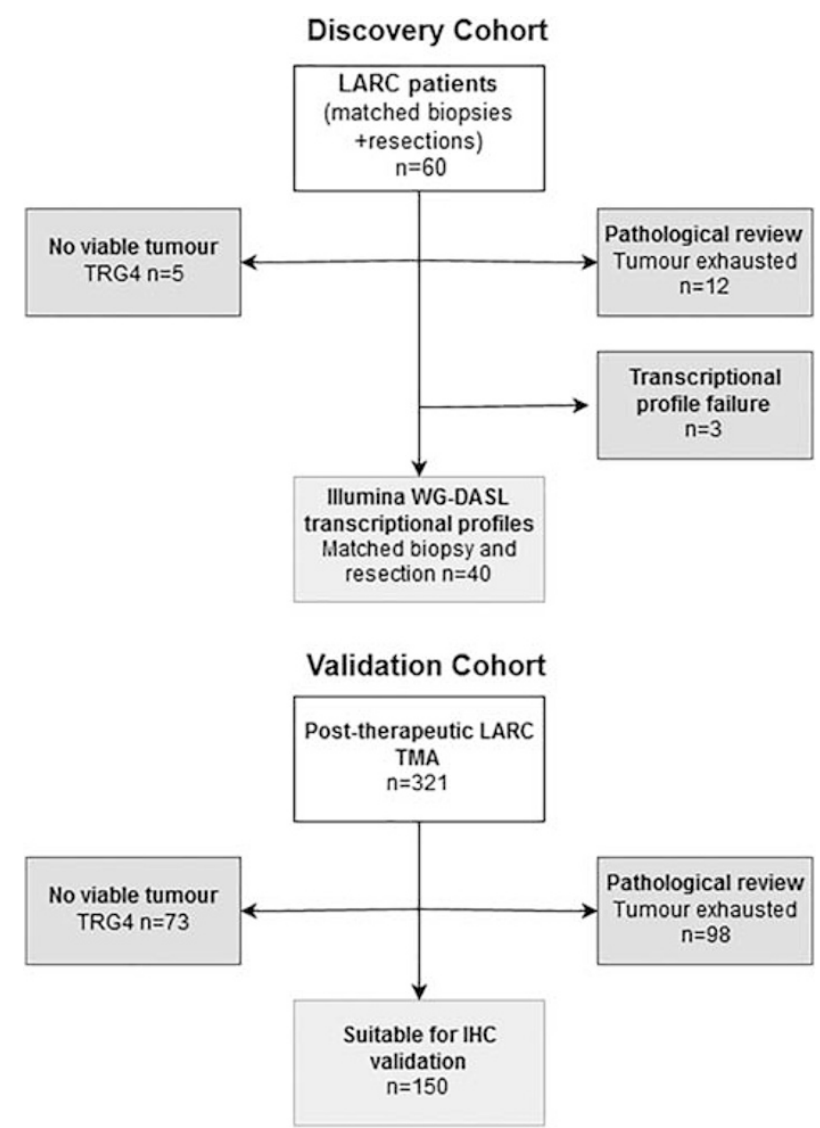

Figure 1 Consort diagram outlining the inclusion and exclusion process for discovery (matched pre-treatment biospies and posttherapeutic resections $n=40$ ) and validation cohort (post-therapeutic tissue microarray $n=150$ ) before data generation and analysis.

discovery cohort between 2004 and 2013 from the Belfast Health and Social Care Trust while the patient specimens from the validation cohort were collected between 2005 and 2011 by NHS Grampian. Only patients who received long-course preoperative chemoradiotherapy consisting of fluoropyrimidines and $45 \mathrm{~Gy}$ pelvic radiation and for whom there was sufficient tumor available for molecular and tissue analysis were included in the study.

\section{Statistical Analysis}

Assessing statistically significant differences between variables was determined using the Student's $t$-test, Log-rank test, Chi-squared and Cox multivariate analysis. Survival was assessed using the KaplanMeier method assessed in SPSSv23 for windows. $P$-values $<0.05$ were considered significant.

\section{High-Throughput Gene Expression Analysis}

The high-throughput gene expression data set was exported using Illumina Genome studio. Data were background corrected and normalized using positive, negative, and housekeeping controls from the 
WG-DASL assay automatically provided by Partek's Report Plug-in. Principal components analysis, differential gene expression analysis (ANOVA between tumor regression grade 1 vs tumor regression grade 3) and hierarchical clustering analysis were conducted using Partek Genomics Suite software, version 6.6 Copyright @; 2016. Differentially expressed genes were determined using fold change thresholds ranging between 2- and 10-fold expression change and only genes with an adjusted $P$ value $<0.05$ (Benjamini-Hochberg) were reported. Heat maps with hierarchical clustering were constructed using Ward's linkage and Euclidean distance. Row expression values were standardized to a mean of zero and scaled to a standard deviation of one. Ingenuity Pathway Analysis and Gene Set Enrichment Analysis (http://software.broadinstitute. org/gsea/index.jsp) were used for biological interpretation of differentially expressed genes.

\section{Next-Generation Sequencing}

Mutational status was acquired from patient biopsies from approximately $10 \mathrm{ng}$ DNA. Sequencing was performed using the Ion Torrent Personal Genome machine, as previously published. ${ }^{12}$ Only $38 / 40$ patients had successful mutational profiles generated.

\section{Defining Microsatellite Instability}

Microsatellite instability was assessed using MSI Analysis system, Version 1.2 (Promega) on the Applied Biosystems 3500 analyzer (ThermoFisher Scientific) using Genemapper v4.1 software. Microsatellite instability was determined by the instability of two or more of the five markers (NR24, BAT26, NR21, BAT25, and MONO27). ${ }^{8}$

\section{Microenvironment Cell Populations Counter}

Microenvironment cell population counter is a peer reviewed software tool that robustly measures the abundance of microenvironment cell populations using transcriptional data generated from heterogeneous tissues. The microenvironment cell population counter R package was downloaded from http:// cit.ligue-cancer.net/?page_id =1243\&lang =en. An estimate score for NK cells, which equates to the absolute abundance of cell type was generated for each NIB13-0103 transcriptional profile. ${ }^{13}$

\section{QUADrATiC Analysis}

The 27 gene signature generated from differential gene expression analysis in Partek Genomics Suite software, version 6.6 Copyright (); (see Supplementary Table 4) was used to perform connectivity map analysis using the QUADrATiC software. ${ }^{14}$ The 27 genes were mapped using annotation files to probe IDs for the Affymetrix HG U133A microarray, as QUADrATiC requires its input signatures in this form. Running the analysis produces a candidate list of positive connections, that is, those FDA-approved compounds within the LINCS database, which appear to induce similar expression patterns to that of the 27 genes, in particular cell lines. This candidate list was further investigated for any compounds with immunemodulating properties.

\section{Results}

Comparative analysis of the discovery and validation cohorts revealed no statistically significant differences observed between the proportions of regression grades (tumor regression grade $3-27.5 \%$ vs $37 \%$, tumor regression grade $2-42.5 \%$ vs $46 \%$, tumor regression grade $1-30 \%$ vs $17 \%$ ), as determined by Chi-squared $(P=0.147)$. This ensured no regression-related sample enrichment or bias in our selected sample sets.

Unsupervised principal components analysis was used to visualize gene expression data from the NIB13-0103 post-therapeutic resection specimens (Figure 2a). The principal components analysis plot highlighted a distinct separation between posttreatment profiles from tumor regression grade 1 and 3 patients with an overlap of tumor regression grade 2 patients with both groups which highlights major differences in gene expression across the patient cohort. Using these tumor transcriptomic profiles, we conducted differential gene expression analysis to identify specific genes of interest for downstream investigation by comparing tumors with either tumor regression grade 1 or tumor regression grade 3 response. Using this supervised approach, we identified 27 genes that had over 10-fold differential expression changes between our contrasting tumor regression grade subgroups. To test the robustness of classifying samples based on tumor regression grade, we used this 27 gene signature generated from contrasting tumor regression grade 1 with tumor regression grade 3 , to classify tumor regression grade 2 tumors. Importantly, we found that our signature clustered tumor regression grade 2 tumors as having intermediate signature expression compared with tumor regression grade 1 and tumor regression grade 3 , indicating a dose response of expression according to tumor regression grade and confirming its potential clinical utility (Figure 2b). The 27 genes consisted of natural killer-cell receptors, cancer/testis antigens, olfactory receptors, and solute carriers (Supplementary Table 4). Ingenuity pathway analysis of the 27 genes suggested that 'Natural Killer Cell signaling' and 'Crosstalk between Dendritic Cells and Natural Killer Cells' are the two defining pathways involved with this tumor regression grade-associated gene list (Figure 2c).

To identify potential FDA-approved compounds that may induce the gene expression phenotype of 
a

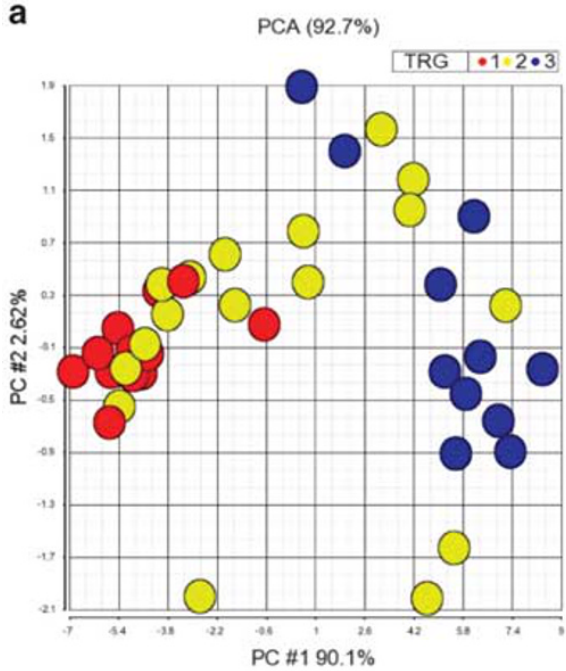

PC \#1 $90.1 \%$ b
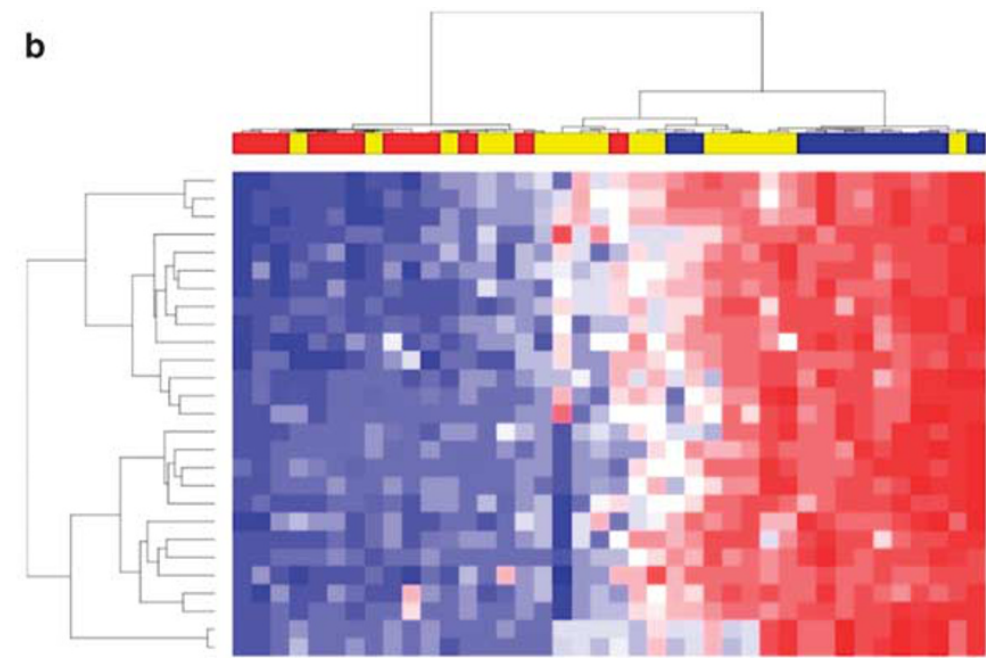

C

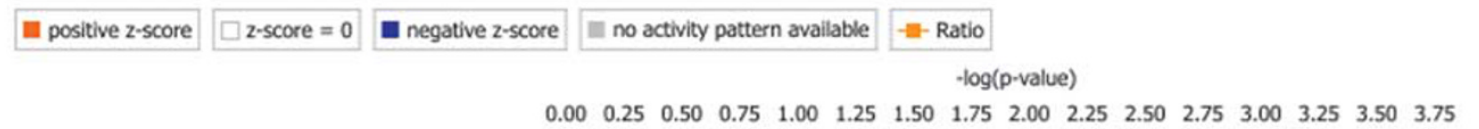

$\begin{array}{llllllllllllllll}0.00 & 0.25 & 0.50 & 0.75 & 1.00 & 1.25 & 1.50 & 1.75 & 2.00 & 2.25 & 2.50 & 2.75 & 3.00 & 3.25 & 3.50 & 3.75\end{array}$

Natural Killer Cell Signaling

Crosstalk between Dendritic Cells and Natural Killer Cells

O 2000-2017 QIAGEN. All rights reserved.

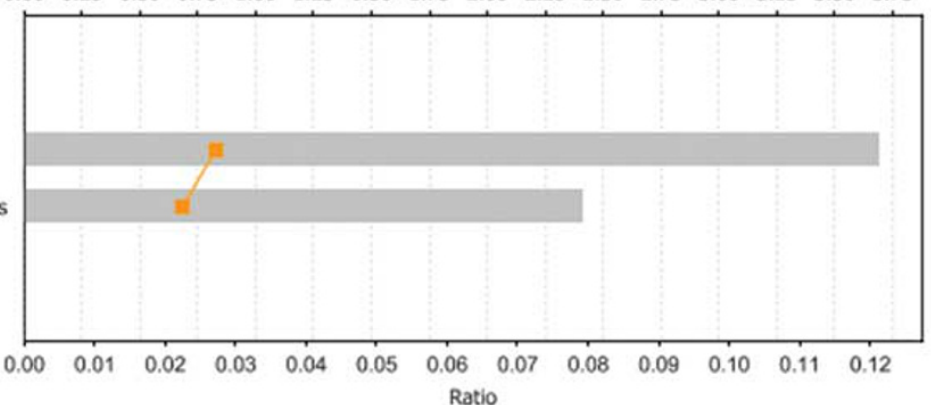

Figure 2 Differential gene expression analysis and pathway analysis of locally advanced rectal cancer resections in discovery cohort $(n=40)$. (a) Principal component analysis of locally advanced rectal cancer resections by tumor regression grade shows separation of tumor regression grade 1 gene expression profiles and tumor regression grade 3 profiles. (b) Heatmap showing hierarchical clustering analysis of resections by 27 most differentially expressed genes, (blue denotes downregulated expression of genes and red upregulation), the heatmap depicts an upregulation of these genes in tumor regression grade 3 resections. (c) Ingenuity pathway analysis of 27 genes. Bar chart shows natural killer-cell signaling to be the most statistically enriched pathway. Legend—tumor regression grade 1 (red), tumor regression grade 2 (yellow) and tumor regression grade 3 (blue).

responders after therapy and harness the good prognosis natural killer-like biology, we utilized the QUADrATiC software. QUADrATiC requires an input of Affymetrix probe IDs. Therefore, the analysis was performed using 21 Affymetrix probe IDs that could be matched from the 27 Illumina probe IDs identified. Statistically significant positive connection scores were generated for the immunomodulatory drug (IMiD) Lenalidomide and the proteosomal inhibitor bortezomib (Supplementary Figure 1a). Both of these agents are already established in routine clinical practice in the treatment of hematological malignancies. ${ }^{15,16}$ The heatmap shows the relative contribution (or Contribution Fraction) of each individual probes input to the overall score and we can conclude from this that the cancer testis antigen families SPANX and GAGE were the most significant contributors to the connection scores and hence are potential targets for the mechanism of action of the two drugs (Supplementary Figure 1a). This result prompted us to cluster resection specimens based on the expression of a list of cancer testis antigens genes. Robust clustering in a tumor regression grade-dependent manner was observed with a higher expression in the responders (tumor regression grade 3) than in tumor regression grade $1 /$ nonresponders (Supplementary Figure 1b). We selected the cancer testis antigen SPANXA1 to validate using RT-PCR. Results indicated that the average fold change between the tumor regression grade 1 group and tumor regression grade 3 patient group was 7.3 (Supplementary Figure 1c). 


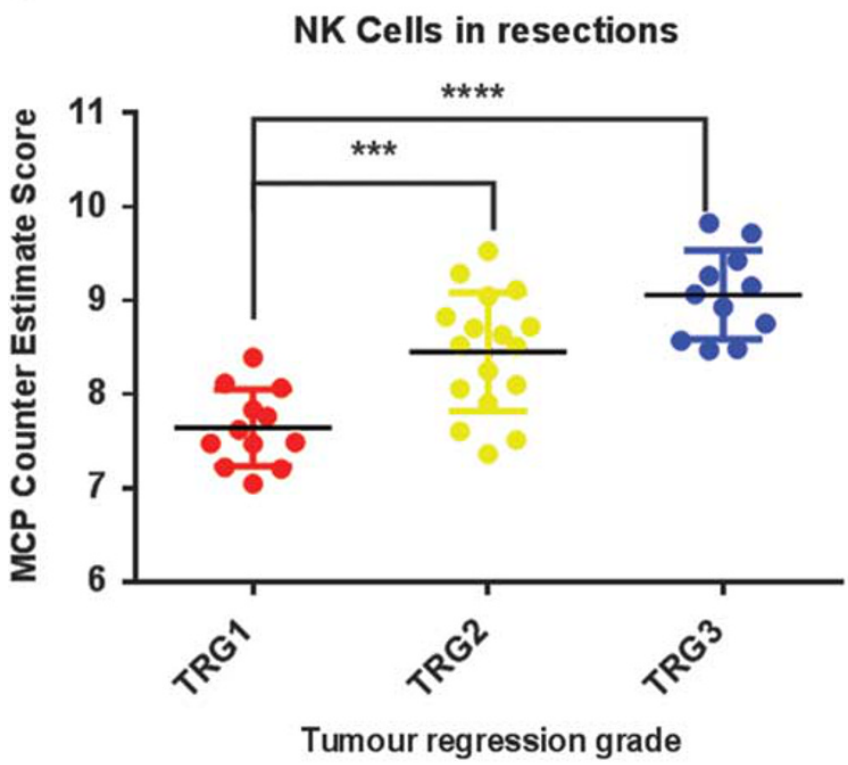

C

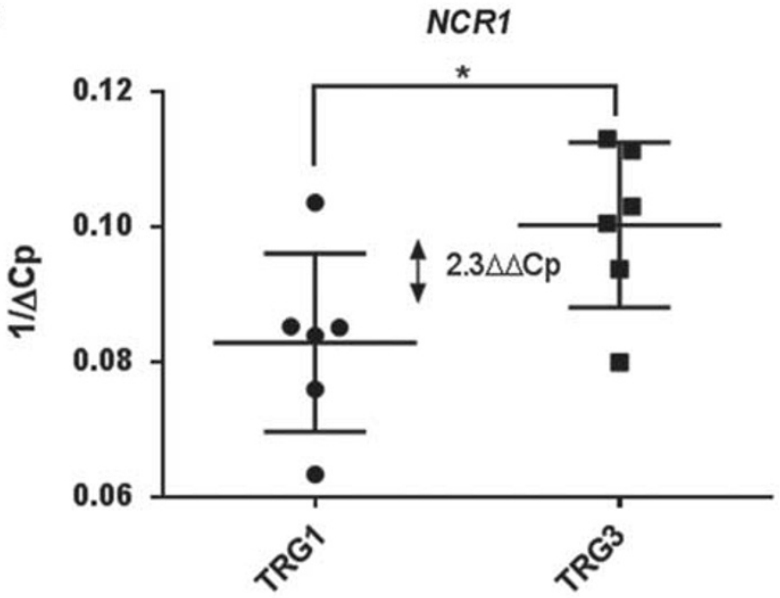

Tumour Regression Grade b
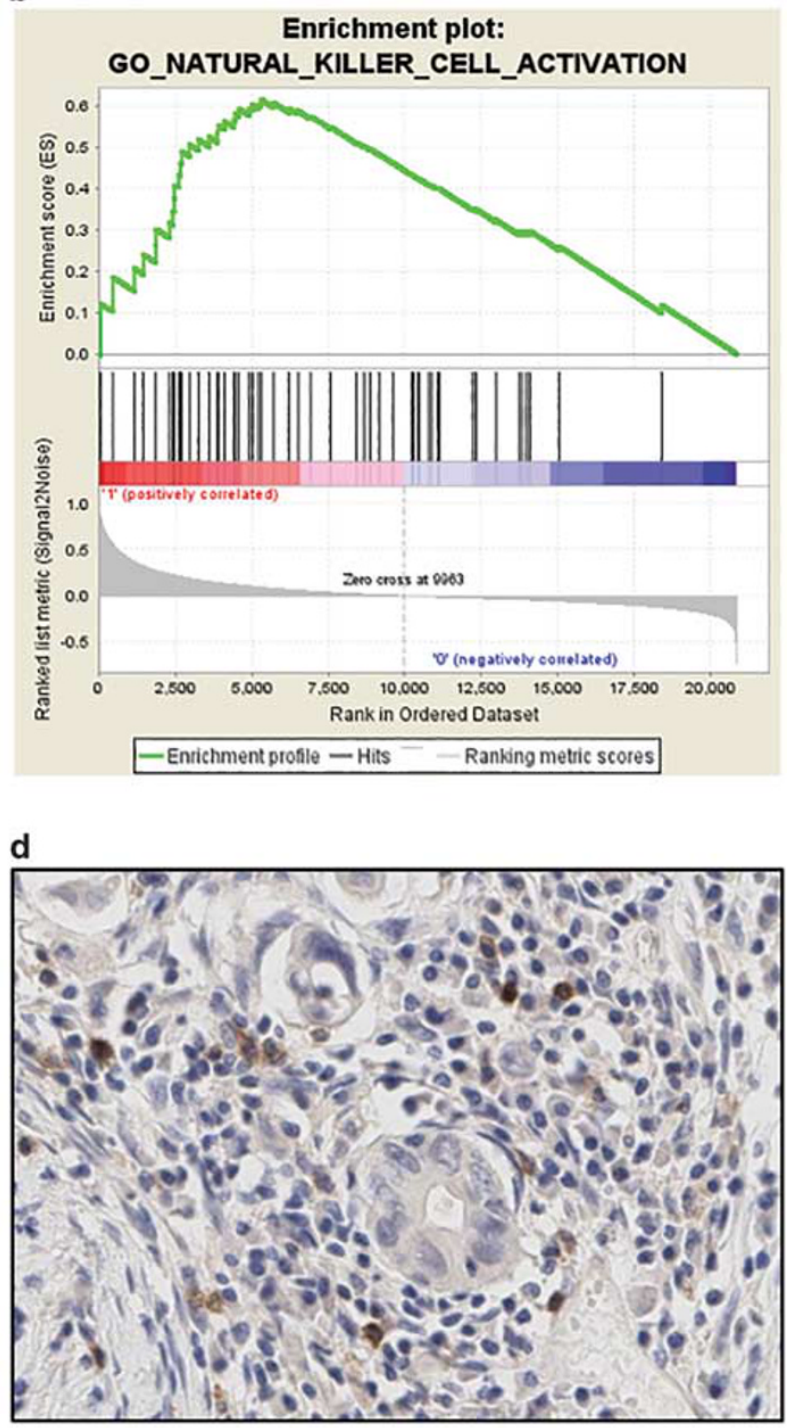

TRG3 resection CD56 IHC

Figure 3 Assessing natural killer-cell biology in locally advanced rectal cancer resections of discovery cohort ( $n=40)$. (a) Microenvironment cell population counter NK cell estimate scores assessed in post-therapeutic locally advanced rectal cancer resections (tumor regression grade 1 vs tumor regression grade $3, P<0.0001$ ) demonstrates a higher abundance of natural killer cells in tumor regression grade 3 resections when compared with tumor regression grade 1. (b) Gene set enrichment analysis comparing tumor regression grade 3 resections (phenotype 1) and tumor regression grade 1 resections (phenotype 0) using the GO_NATURAL KILLER_CELL_ACTIVATION gene set (FDR $<0.0001$ ), shows an upregulation of genes associated with natural killer-cell activity in tumor regression grade 3 resections compared with tumor regression grade 1 (c) RT-PCR validation of mRNA expression of NCR1 in tumor regression grade 1 +tumor regression grade 3 rectal resections. $(P=0.04$, Student's $t$-test). (d) Image depicting CD56-positive natural killer cells by immunohistochemistry in tumor regression grade 3 post-therapeutic resection from the discovery cohort $\left(\times 40\right.$ magnification). ${ }^{*} 0.0143$, $* * * 0.0007$ and $* * * *<0.0001$.

Based on our observations, microenvironment cell population counter was used to assess the abundance of natural killer cells in our resection specimens, where we observed significantly increased natural killer-cell populations in a tumor regression grade-dependent manner (Figures 3a, $P<0.0001$, Tukey's multiple comparisons test). This finding was complemented by gene set enrichment analysis comparing tumor regression grade 3 vs tumor regression grade 1 resection transcriptional profiles, which showed a statistically significant upregulation of the "GO_NATURAL_KILLER_CELL_ACTIVATION" gene set in tumor regression grade 3 resections (Figure 3b). Subsequently, validation of the natural killer-cell-specific marker NCR1, which emerged as a differentially expressed gene was performed using RT-PCR. There was observed to be a statistically significant 2.3-fold difference in expression between tumor regression grade 3 and tumor regression grade 1 patients (Figure 3c, 
$P=0.04)$. The cell surface protein NCAM1 also known as CD56 is considered one of the most specific proteomic markers for natural killer cells. A number of remaining resections with viable tumor from the discovery cohort were stained by immunohistochemistry for the natural killer-cell marker CD56, confirming the presence of CD56-positive cells in a tumor regression grade 3 resection (Figure 3d). Interestingly, NCAM1 is considered a poor marker of natural killer cells at the transcript level due to its nonspecific expression, as it is also strongly expressed by nerve ganglion and for this reason it is not included as a marker of natural killercell quantification in microenvironment cell population counter. ${ }^{13}$ Supplementary Figure 2 supports this finding as it was observed that the expression of NCAM1 is not tumor regression grade-dependent in our cohort unlike the natural killer-cell marker NCR1.

We next examined the transcriptional profiles obtained for the matched pre-treatment biopsies and assessed the abundance of natural killer cells, again using microenvironment cell population counter. It was observed that the presence of natural killer cells in pre-treatment biopsies was not tumor regression grade-dependent and therefore did not have predictive value (Figure 4a). A trend was observed that the abundance of natural killer cells tended to increase in tumor regression grade 3 patients in a matched pre- and post-analysis while tumor regression grade 1 patients experienced increases and decreases in the abundance of natural killer cells, however this did not reach statistical significance (Figures $4 \mathrm{~b}$ and $\mathrm{c}$ ).

Next-generation sequencing analysis of clinically relevant mutations (KRAS, TP53, BRAF, NRAS, APC, ERBB2, PIK3CA, and SMAD4) was performed to assess any association with mutational profile and the presence of our natural killer-like gene expression signature. There was no observable association between our mutational data and the tumor regression grade- dependent transcriptional subgroups identified, which is highlighted by a heatmap that depicts the mutations present in each tumor regression grade (Supplementary Figure 3). We also performed a comparative analysis to identify differences in the mutational landscape of the NIB13-0103 rectal cohort $(n=38)$ when compared with the TCGA, Nature 2012 colorectal cancer data set. ${ }^{17,18}$ The data set was filtered for rectal cancer stage II-III $(n=31)$ using cBioPortal. This comparison of mutations in the NIB13-0103 and TCGA cohorts demonstrated no statistical difference in the proportions of mutations of TP53 $(73.68 \%$ vs $80.65 \%, P=0.547)$, KRAS $(55.26 \%$ vs $45.16 .0 \% P=0.472)$, APC $(39.47 \%$ VS $77.42 .0 \% \quad P=0.283)$, NRAS $(0 \%$ vS $6.45 \%$ $P=0.198)$, BRAF $(2.63 \%$ vs $0 \% P=1.0)$, ERBB2 $(0 \%$ vs $\quad 6.45 \% \quad P=0.198)$, PIK3CA $(5.26 \%$ vs $9.68 \%$ $P=0.651)$, SMAD4 $(7.89 \%$ vs $3.23 \% P=0.622) .{ }^{17,18}$ However, interpretation of these differences is difficult considering the small sample size of both
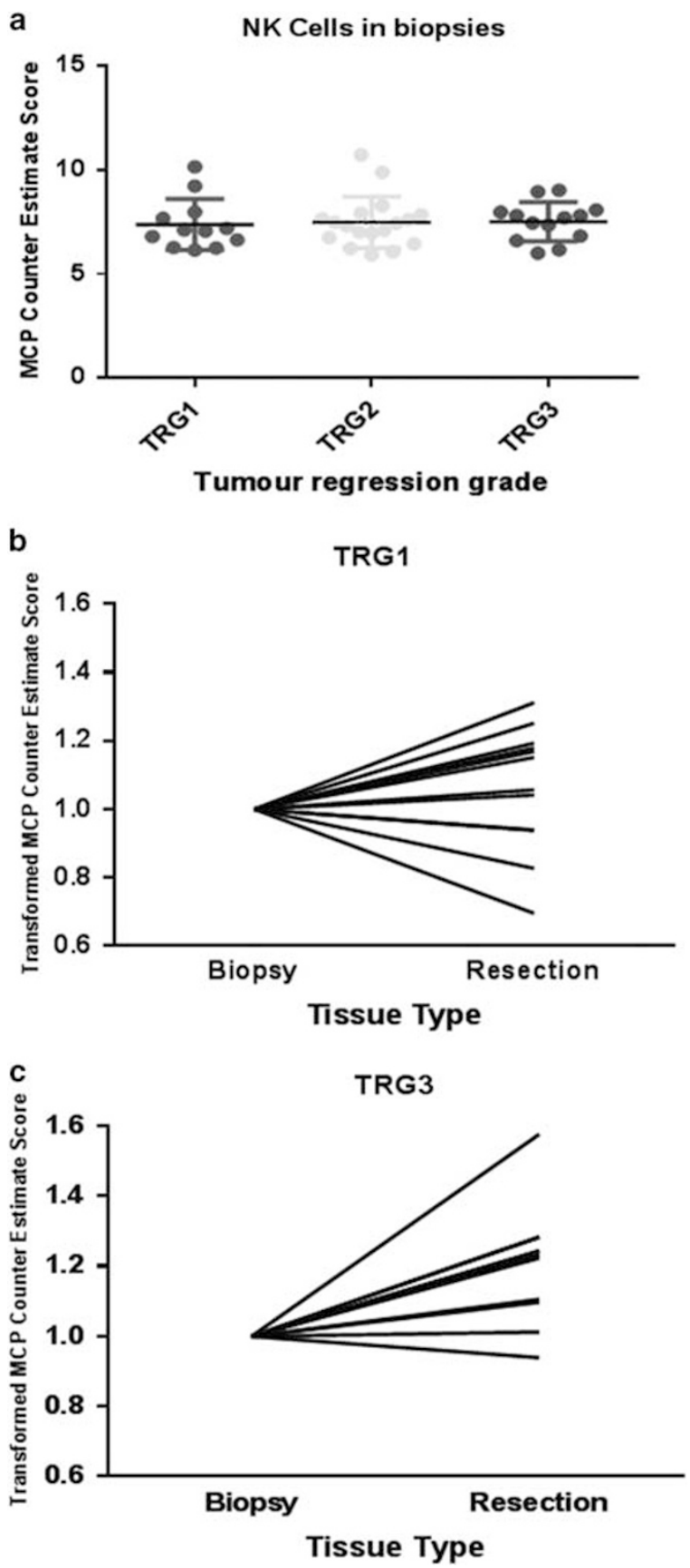

Figure 4 Assessing the abundance of NK cells in biopsies and matched resections in discovery cohort $(n=40)$ using microenvironment cell population counter. (a) Microenvironment cell population counter NK cell estimate scores assessed in pre-therapeutic locally advanced rectal cancer biopsies shows no difference in the abundance of natural killer cells between tumor regression grades (b) Normalized microenvironment cell population counter NK cell estimate scores assessed in pre-therapeutic tumor regression grade 1 locally advanced rectal cancer biopsies and matched resections shows changes after long-course preoperative chemoradiotherapy (c) Normalized microenvironment cell population counter NK cell estimate scores assessed in pre-therapeutic tumor regression grade 3 locally advanced rectal cancer biopsies and matched resections shows changes after long-course preoperative chemoradiotherapy. 
a

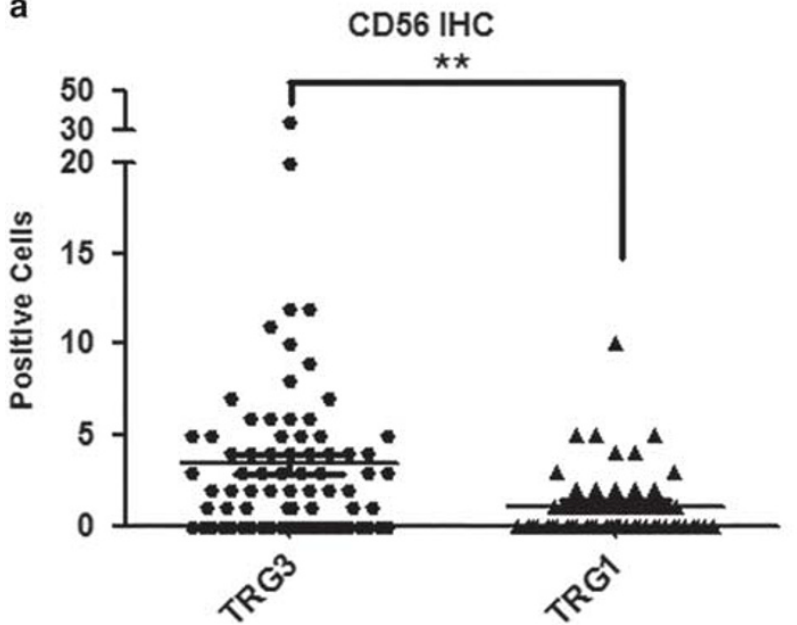

Tumour regression grade b TRG3 CD56 positive

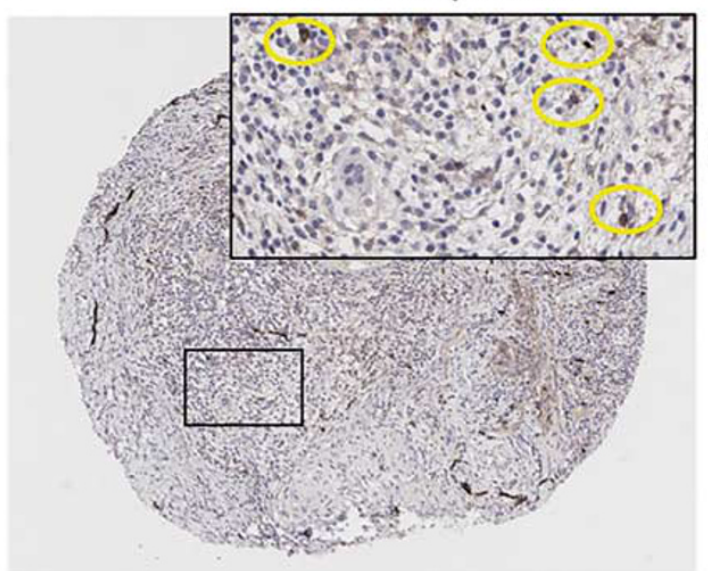

X40 Mag

C TRG1 CD56 positive

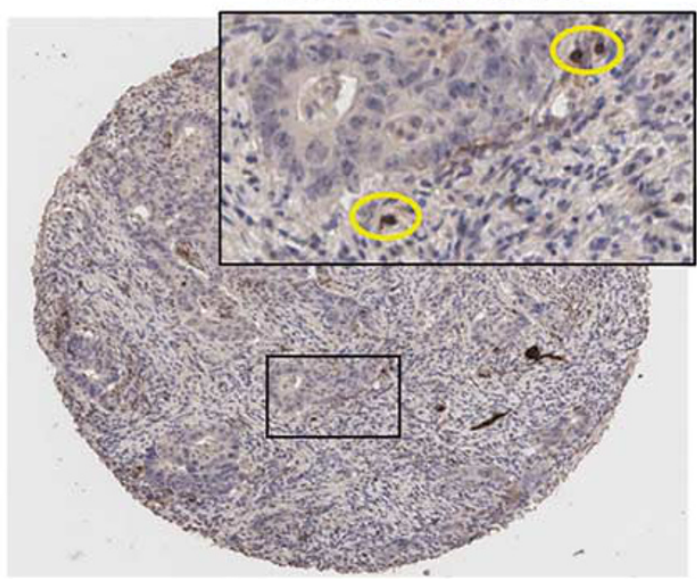

X40 Mag

X5 Mag

Figure 5 The association between CD56 positive natural killer cells and tumor regression grade in post-therapeutic validation locally advanced rectal cancer tissue microarray $(n=150)$ using immunohistochemistry. (a) Dot plot showing total counts of CD56 positive natural killer cells per tissue microarray core grouped by tumor regression grading (tumor regression grade 3 vs tumor regression grade 1). There was observed to be significantly more CD56 positive natural killer cells in tumor regression grade 3 resections compared with tumor regression grade 1 resections by Student's $t$-test $P=0.0083$. (b) Representative image of a CD56-positive immunohistochemistry in tissue microarray core of a tumor regression grade 3 responder with CD56 natural killer cells highlighted in yellow ( $\times 40$ magnification). (c) Representative image of a CD56-positive tissue microarray core by immunohistochemistry of a tumor regression grade 1 responder with CD56 natural killer cells highlighted in yellow ( $\times 40$ magnification).

cohorts. Similarly, we could not determine an association between MSI status in our patients and the natural killer-like response as no tumors were MSI high.

To confirm the gene expression findings using a diagnostically relevant methodology, we performed an immunohistochemistry-based assessment of resection tumor samples from an independent rectal cancer cohort $(n=150)$ using the NK marker CD56. We observed a significantly higher total number of CD56+ cells per core in the tumor regression grade 3 resections compared with the number of CD56+ cells in tumor regression grade 1 resection specimens $(P$-value $=0.008$; Figures $5 \mathrm{a}-\mathrm{C})$. The prognostic significance of CD56 positivity was investigated in this independent cohort, with patients categorized as CD56 positive when $\geq 4$ positive cells per core were observed. Using this threshold for positivity, patients with CD56 positivity were shown to have a significantly better overall survival $(\mathrm{HR}=0.282,95 \% \mathrm{CI}=$ $0.109-0.729, \chi^{2}=7.854, P=0.005$ ), which was independent of tumor regression grading (Figure 6a). Interestingly CD56 positivity conferred a trend towards better outcomes within all tumor regression grades, particularly within the tumor regression grade 1 samples within the independent cohort (Figure 6b-d). Furthermore, CD56 positivity was shown to be independently prognostic through multivariate analysis $P<0.006$ (Supplementary Table 5).

\section{Discussion}

It has been proposed that common alterations to immune/inflammatory pathways may confer sensitivity to long-course preoperative chemoradiotherapy 


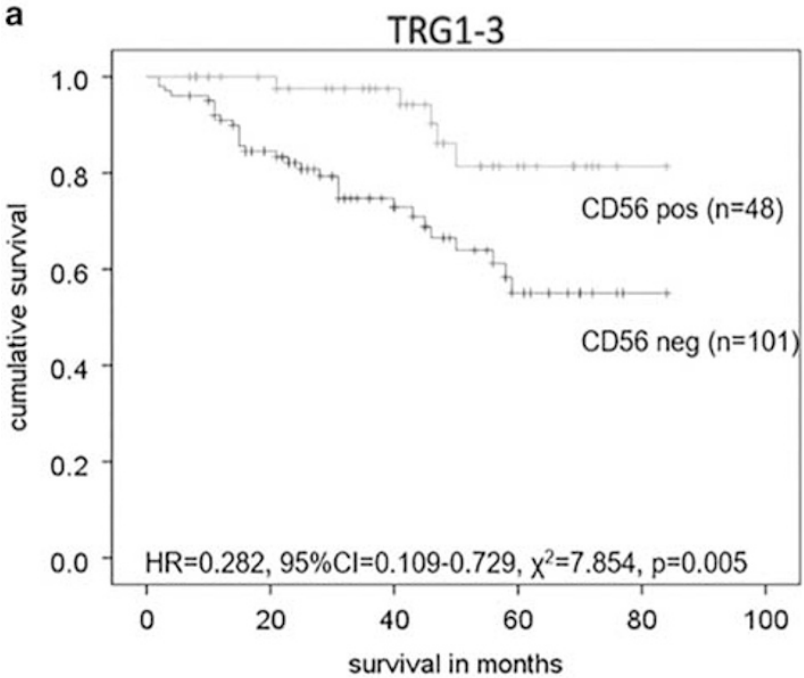

C

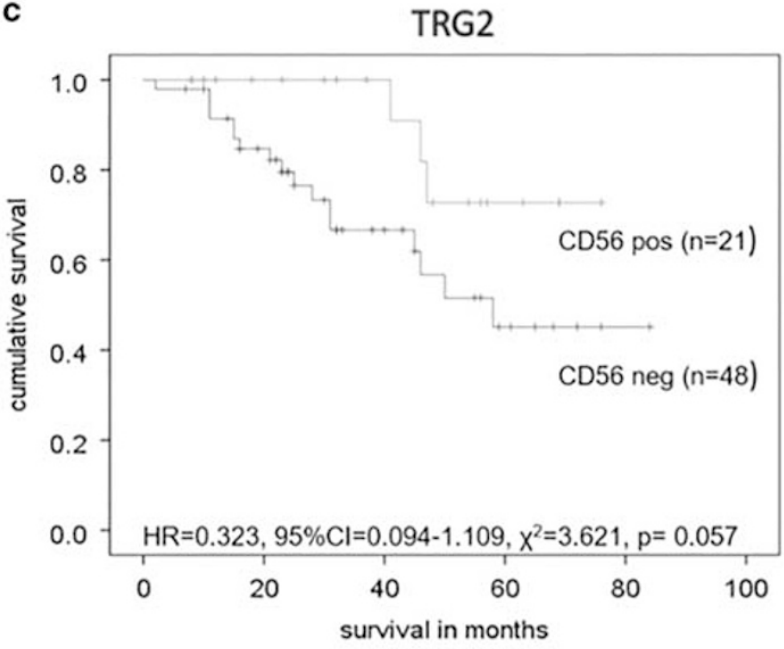

b

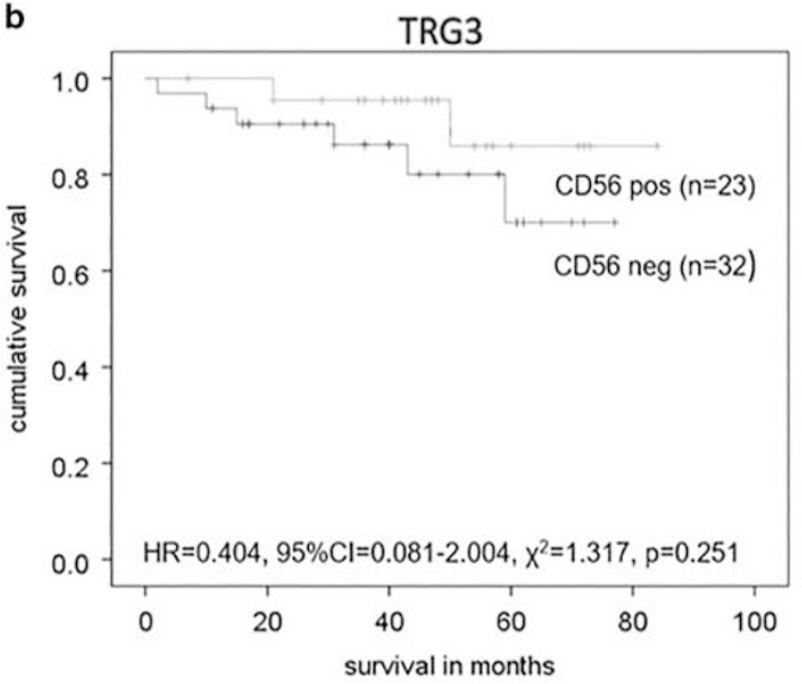

d

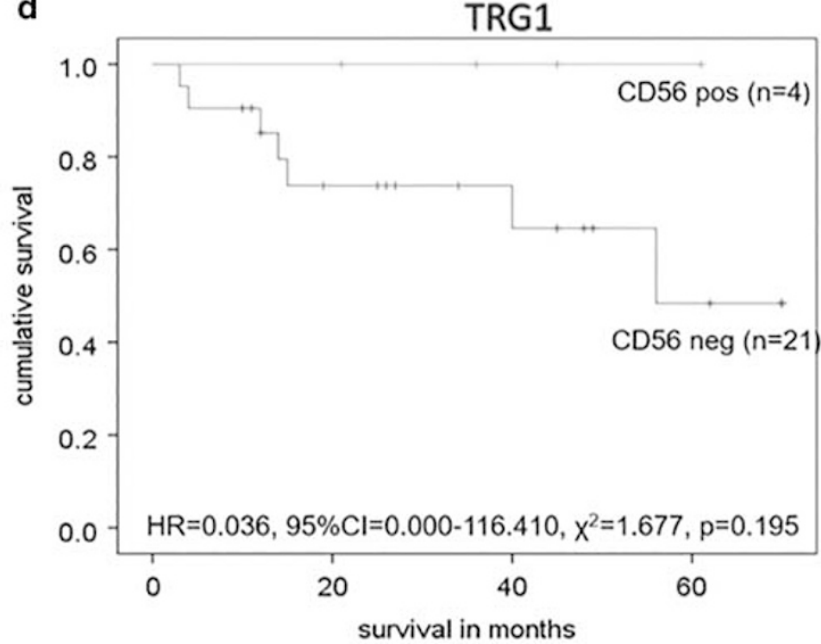

Figure 6 Investigating the influence of CD56 immunohistochemistry positivity on overall survival in post-therapeutic locally advanced rectal cancer validation tissue microarray $(n=149)^{*}$. (a) Kaplan-Meier curves showing overall survival of all patients (tumor regression grade $1-3$ ) based on CD56 positivity ( $\mathrm{HR}=0.282,95 \% \mathrm{CI}=0.109-0.729, \chi^{2}=7.854$, log-rank $P=0.005$ ). (b) Kaplan-Meier curves showing overall survival of CD56-positive patients within tumor regression grade 3 subgroup $\left(\mathrm{HR}=0.404,95 \% \mathrm{CI}=0.081-2.004, \chi^{2}=1.317\right.$, $\log$-rank $P=0.251$ ). (c) Kaplan-Meier curves showing overall survival of CD56-positive patients within tumor regression grade 2 subgroup $\left(\mathrm{HR}=0.323,95 \% \mathrm{CI}=0.094-1.109, \chi^{2}=3.621\right.$, log-rank $\left.P=0.057\right)(\mathrm{d})$ Kaplan-Meier curves showing overall survival of CD56-positive patients within tumor regression grade 1 subgroup $\left(\mathrm{HR}=0.036,95 \% \mathrm{CI}=0.000-116.410, \chi^{2}=1.677, \log\right.$-rank $\left.P=0.195\right)$. ${ }^{*}$ One patient from CD56 immunohistochemistry validation tissue microarray did not have survival data.

in locally advanced rectal cancer patients. ${ }^{19}$ Understanding the nature of the immune response that occurs following long-course preoperative chemoradiotherapy may be problematic in pre-therapeutic biopsies. Therefore, we have interrogated posttherapeutic locally advanced rectal cancer resections using a combination of modern high-throughput genomic technologies and traditional pathology techniques, to characterize the immune response that has occurred and allow us to hypothesize its use as a predictive biomarker in on-treatment biopsies and also how to harness it for therapeutic benefit.

Both radiation and chemotherapy can augment immune response possibly through increased expression of antigens, ${ }^{8}$ these antigens may be responsible for engaging the cytotoxic cells of the adaptive immune system. ${ }^{9-11}$ The increased presence of CD8+ cytotoxic lymphocytes are well defined in colorectal cancer particularly in microsatellite unstable tumors and are associated with an improved prognosis. ${ }^{12}$ Based on the expression of immune checkpoints such as PDL1, there is rationale for the use of immune checkpoint inhibitors in MSI$\mathrm{H}$ colorectal cancers and although most immune checkpoint inhibitors execute their effects through CD8+ cytotoxic lymphocytes, therapeutic modulation of other immune cell types such as macrophages, myeloid-derived suppressor cells and natural killer cells could have similar effects in the rectal paradigm. ${ }^{13-15}$ Natural killer cells are 
cytotoxic lymphocytes of the innate immune system and act as the body's first line of defence against tumor cells. Natural killer cells have been observed to be critical for response to gemcitabine in pancreatic mouse models. ${ }^{16}$ Furthermore, the presence and activity of natural killer cells may be an indicator of favorable outcome in breast cancer and oropharyngeal squamous cell carcinoma. ${ }^{17,18}$

In this study, we observed significantly increased expression of genes associated with a natural killerlike phenotype after long-course preoperative chemoradiotherapy. This, to our knowledge, is a novel observation following treatment of locally advanced rectal cancer. Although the overall frequency of these cells may not be very high, they do appear to be associated with a strong biological response underpinning their potential biological significance and implications in tumor regression. The presence of natural killer cells was initially defined in silico using the microenvironment cell population counter tool, which gives a robust estimation to the abundance of NK cells. ${ }^{13}$ The abundance of natural killer cells was also assessed in the pre-therapeutic biopsies, however, increased natural killer cells was shown to be therapy related and therefore not a predictive biomarker of response. Validation of the microarray results was performed by using RT-PCR using NCR1. The expression of NCR1 is considered one of the more specific markers of natural killer cells, however, natural cytotoxicity receptors can also be expressed on innate lymphoid cells. $^{20,21}$ The delineation of classical natural killer-cell populations from iNKT cells, mucosal associated invariant $\mathrm{T}$ cells, ILC1s, ILC3s, and gamma delta $\mathrm{T}$ cells remains challenging in formalin-fixed paraffin-embedded tissue as there is considerable overlap in the receptor repertoires, and as such cell-specific detailing still relies on flow cytometry approaches. ${ }^{22-26}$ Therefore, the general expression of immune-derived CD56 still remains the gold standard for detecting natural killer cells in formalin-fixed paraffin-embedded tissue. As a result of our gene expression profiling, we investigated the expression of the natural killer-cell marker CD56 using immunohistochemistry. A comprehensive analysis of CD56 natural killer-cell expression of post-therapeutic resection specimens could not be performed in the discovery cohort due to tumor exhaustion in many of the tumor regression grade 3 specimens through serial sectioning of the formalinfixed paraffin-embedded blocks. However, CD56 immunohistochemistry was successfully used in the validation cohort and we observed this cell type to be more frequent in tumor regression grade 3 responders than tumor regression grade 1 after therapy. Our evaluation of CD56 immunohistochemistry was performed on tissue microarray cores, which is a limitation of this study due to potential regional bias, therefore, we feel that further adjusting our scoring criterion to enable CD56 assessment in fullresection specimens deserves further investigation.
The presence of CD56+ natural killer cells by immunohistochemistry in treatment naive colorectal cancer has been described as infrequent; ${ }^{27,28}$ on this basis, we did not discern between those in the adjacent-stroma and intraepithelial natural killer cells, however, in a larger data set of full face resection specimens, we would prefer to assess the influence of different regional infiltration.

A recent study demonstrated the clinical importance of natural killer cells in colorectal cancer, Sconocchia et al. ${ }^{10}$ observed that an enrichment of both natural killer and CD8 $\mathrm{T}$ cells improved the outcome of colorectal cancer patients. With regards to post-therapeutic infiltration of immune cells in rectal cancer, the presence of natural killer cells has not previously been observed to be significant in post-therapy rectal specimens by CD56+ immunohistochemistry. ${ }^{7}$ However, it is worth noting in this study, positive infiltration of CD56 natural killer cells was assessed as $4>$ /high-powered field rather than $\geq 4$ positive cells per core, which may account for different observations. Post-therapeutic infiltration of adaptive immune cells in locally advanced rectal cancer has been shown to increase after therapy, Teng et al observed significantly higher CD3 and CD8 TILs and in a further study also CD4 tumor-infiltrating lymphocytes. ${ }^{6,7}$

It is evident that the clinical utility of measuring natural killer-cell density in pre-treatment locally advanced rectal cancer biopsies may be limited. However, our observation of tumor regression grade-dependent natural killer-cell abundance in post-therapeutic resections warrants the collection and analysis of on-treatment or post-therapeutic biopsies to assess natural killer-cell biology that may have the potential to provide real-time clinical guidance on the efficacy of the therapeutic intervention. We observed that patients with CD56 positivity had a significantly better outcome to chemoradiation than those with lower levels of CD56 staining with multivariate analysis showing CD56 positivity to be a significant independent prognostic factor. As a result, we hypothesize that encouraging this natural killer-cell biology with immune-modulating compounds may be a novel therapeutic strategy for locally advanced rectal cancer patients.

By using QUADrATiC software to carry out connectivity mapping analysis against the subset of FDA-approved compounds in the LINCs database, we identified lenalidomide and bortezomib as two FDA-approved compounds with proven antitumor and immunomodulatory effects that may induce the expression of the 27 genes identified in Supplementary Table 4. Lenalidomide and proteosomal inhibitors (bortezomib and carfilzomib) are already in clinical use for the treatment of hematological malignancies and have demonstrated the ability regulate natural killer-cell function. ${ }^{15,16,29-33}$ By analyzing the connections further using the Contribution Fraction capabilities of QUADrATiC, 
both compounds were shown to have an association with the increased expression of cancer testis/ antigens which are potentially activators of $\mathrm{T}$ cell receptor-mediated cytotoxicity, and thus may have a role in therapy-related immunomodulation..$^{4,5}$

We hypothesize that using immune-modulating compounds such as lenalidomide and low-dose bortezomib (or the second-generation proteosomal inhibitor Carfilzomib) as single agents or even in combination could prime the natural killer-cellrelated immune system, improving the efficacy of standard chemoradiotherapy. Aside from immunemodulating compounds such as bortezomib and lenalidomide, there are currently three other methods of stimulating or harnessing natural killer-cell function (i) endogenous interleukins such as IL-2 and IL-15, can stimulate natural killer-cell activity, ${ }^{34}$ (ii) monoclonal antibodies that act as natural killercell immune checkpoint inhibitors such as lirilumab, ${ }^{35}$ (iii) autologous and allogeneic transfer of wild-type and genetically engineered natural killer cells are also being tested for efficacy in both hematological and solid tumors. ${ }^{36-38}$

In conclusion, we have provided independently validated evidence that the presence of a CD56+ natural killer-like immune response after therapy confers better overall survival for locally advanced rectal cancer patients, which may also be independent of tumor regression. We can conclude that natural killer cells are an important element of the immune landscape of locally advanced rectal cancer and on this basis we have hypothesized that harnessing a natural killer-cell response using immune-modulating compounds could further improve outcomes for patients with locally advanced rectal cancer. Finally, real-time longitudinal assessment of the natural killer-cell response in ontreatment biopsy material may inform clinical decision-making for duration of treatment or surgical decision to remove tumor if no response to longcourse preoperative chemoradiotherapy is evident.

\section{Acknowledgments}

This work was supported by a very generous grant from the Sean Crummey Memorial Fund. The staff and infrastructure provided by the N. Ireland Biobank and the Belfast Experimental Cancer Medicine Centre allowed this research to take place. These are supported by the Research and Development Division of the N. Ireland Public Health Agency and Cancer Research UK. We also express our gratitude to the staff at the Grampian Biorepository for providing the Tissue microarray materials for validation purposes.

\section{Disclosure/conflict of interest}

The authors declare no conflict of interest.

\section{References}

1 Gollins S, Sebag-Montefiore D. Neoadjuvant treatment strategies for locally advanced rectal cancer. Clin Oncol (R Coll Radiol) 2016;28:146-151.

2 Trakarnsanga A, Gonen M, Shia J et al. Comparison of tumor regression grade systems for locally advanced rectal cancer after multimodality treatment. J Natl Cancer Inst 2014;106:dju248.

3 Rodel C, Martus P, Papadoupolos T, et al. Prognostic significance of tumor regression after preoperative chemoradiotherapy for rectal cancer. J Clin Oncol 2005;23:8688-8696.

4 Sharma A, Bode B, Wenger RH, et al. gamma-Radiation promotes immunological recognition of cancer cells through increased expression of cancer-testis antigens in vitro and in vivo. PLoS ONE 2011;6:e28217.

5 Sharma A, Bode B, Studer G, et al. Radiotherapy of human sarcoma promotes an intratumoral immune effector signature. Clin Cancer Res 2013;19:4843-4853.

6 Teng $\mathrm{F}, \mathrm{Mu} \mathrm{D}$, Meng $\mathrm{X}$, et al. Tumor infiltrating lymphocytes (TILs) before and after neoadjuvant chemoradiotherapy and its clinical utility for rectal cancer. Am J Cancer Res 2015;5:2064-2074.

7 Teng F, Meng X, Kong L, et al. Tumor-infiltrating lymphocytes, forkhead box P3, programmed death ligand-1, and cytotoxic $\mathrm{T}$ lymphocyte-associated antigen-4 expressions before and after neoadjuvant chemoradiation in rectal cancer. Transl Res 2015;166: 721-732.e1.

8 Alvi MA, McArt DG, Kelly P, et al. Comprehensive molecular pathology analysis of small bowel adenocarcinoma reveals novel targets with potential for clinical utility. Oncotarget 2015;6:20863-20874.

9 Luu-The V, Paquet N, Calvo E, et al. Improved real-time RT-PCR method for high-throughput measurements using second derivative calculation and double correction. Biotechniques 2005;38:287-293.

10 Sconocchia G, Eppenberger S, Spagnoli GC, et al. NK cells and T cells cooperate during the clinical course of colorectal cancer. Oncoimmunology 2014;3:e952197.

11 Brown GT, Cash B, Alnabulsi A, et al. The expression and prognostic significance of bcl-2-associated transcription factor 1 in rectal cancer following neoadjuvant therapy. Histopathology 2016;68:556-566.

12 McCourt CM, McArt DG, Mills K, et al. Validation of next generation sequencing technologies in comparison to current diagnostic gold standards for BRAF, EGFR and KRAS mutational analysis. PLoS ONE 2013;8: e69604.

13 Becht E, Giraldo NA, Lacroix L, et al. Estimating the population abundance of tissue-infiltrating immune and stromal cell populations using gene expression. Genome Biol 2016;17:218.

14 O'Reilly PG, Wen Q, Bankhead P, et al. QUADrATiC: scalable gene expression connectivity mapping for repurposing FDA-approved therapeutics. BMC Bioinformatics 2016;17:198-016-1062-1.

15 Totani H, Ri M, Kato C, et al. Phase I study of once weekly treatment with bortezomib in combination with lenalidomide and dexamethasone for relapsed or refractory multiple myeloma. Int J Hematol 2016;103:316-321.

16 Wang $M$, Martin $\mathrm{T}$, Bensinger $\mathrm{W}$, et al. Phase 2 dose-expansion study (PX-171-006) of carfilzomib, lenalidomide, and low-dose dexamethasone in relapsed or progressive multiple myeloma. Blood 2013; 122:3122-3128. 
17 Cerami E, Gao J, Dogrusoz U, et al. The cBio cancer genomics portal: an open platform for exploring multidimensional cancer genomics data. Cancer Discov 2012;2:401-404.

18 Gao J, Aksoy BA, Dogrusoz U, et al. Integrative analysis of complex cancer genomics and clinical profiles using the cBioPortal. Sci Signal 2013;6:pl1.

19 Agostini M, Janssen KP, Kim IJ, et al. An integrative approach for the identification of prognostic and predictive biomarkers in rectal cancer. Oncotarget 2015;6:32561-32574.

20 Artis D, Spits H. The biology of innate lymphoid cells. Nature 2015;517:293-301.

21 Morvan MG, Lanier LL. NK cells and cancer: you can teach innate cells new tricks. Nat Rev Cancer 2015;16: 7-19.

22 Bjorkstrom NK, Ljunggren HG, Sandberg JK. CD56 negative NK cells: origin, function, and role in chronic viral disease. Trends Immunol 2010;31:401-406.

23 Godfrey DI, Uldrich AP, McCluskey J, et al. The burgeoning family of unconventional $\mathrm{T}$ cells. Nat Immunol 2015;16:1114-1123.

24 Gapin L. Where do MAIT cells fit in the family of unconventional T cells? PLoS Biol 2009;7:e70.

25 Spits H, Bernink JH, Lanier L. NK cells and type 1 innate lymphoid cells: partners in host defense. Nat Immunol 2016;17:758-764.

26 Killig M, Glatzer T, Romagnani C. Recognition strategies of group 3 innate lymphoid cells. Front Immunol 2014;5:142.

27 Sandel MH, Speetjens FM, Menon AG, et al. Natural killer cells infiltrating colorectal cancer and MHC class I expression. Mol Immunol 2005;42:541-546.

28 Halama N, Braun M, Kahlert C, et al. Natural killer cells are scarce in colorectal carcinoma tissue despite high levels of chemokines and cytokines. Clin Cancer Res 2011;17:678-689.
29 Armeanu S, Krusch M, Baltz KM, et al. Direct and natural killer cell-mediated antitumor effects of lowdose bortezomib in hepatocellular carcinoma. Clin Cancer Res 2008;14:3520-3528.

$30 \mathrm{Hu}$ W, Gao ZY, Wang W. Bortezomib enhances the sensitivity of prostate cancer cells to natural killer cellmediated cytotoxicity. Zhonghua Nan Ke Xue 2014;20: 218-224.

$31 \mathrm{Wu} \mathrm{L}$, Parton A, Lu L, et al. Lenalidomide enhances antibody-dependent cellular cytotoxicity of solid tumor cells in vitro: influence of host immune and tumor markers. Cancer Immunol Immunother 2011;60:61-73.

$32 \mathrm{Xu} \mathrm{Y,} \mathrm{Sun} \mathrm{J,} \mathrm{Sheard} \mathrm{MA,} \mathrm{et} \mathrm{al.} \mathrm{Lenalidomide} \mathrm{over-}$ comes suppression of human natural killer cell antitumor functions by neuroblastoma microenvironmentassociated IL-6 and TGFbeta1. Cancer Immunol Immunother 2013;62:1637-1648.

33 Krieg S, Ullrich E. Novel immune modulators used in hematology: impact on NK cells. Front Immunol 2013;3:388.

34 Childs RW, Carlsten M. Therapeutic approaches to enhance natural killer cell cytotoxicity against cancer: the force awakens. Nat Rev Drug Discov 2015;14:487-498.

35 Kohrt HE, Thielens A, Marabelle A, et al. Anti-KIR antibody enhancement of anti-lymphoma activity of natural killer cells as monotherapy and in combination with anti-CD20 antibodies. Blood 2014;123:678-686.

36 Lim O, Jung MY, Hwang YK, et al. Present and future of allogeneic natural killer cell therapy. Front Immunol 2015;6:286.

37 Rezvani K, Rouce RH. The application of natural killer cell immunotherapy for the treatment of cancer. Front Immunol 2015;6:578.

38 Burga RA, Nguyen T, Zulovich J, et al. Improving efficacy of cancer immunotherapy by genetic modification of natural killer cells. Cytotherapy 2016;18: 1410-1421.

Supplementary Information accompanies the paper on Modern Pathology website (http://www.nature.com/ modpathol) 